\title{
Reactions of the vicinal dianion of di-(-)-menthyl succinate with carbonyl compounds and benzyl bromide
}

\author{
Darunee Soorukram,* Sariya Yodwaree, Patoomratana Tuchinda, Chutima Kuhakarn, \\ Vichai Reutrakul, and Manat Pohmakotr \\ Department of Chemistry and Center of Excellence for Innovation in Chemistry (PERCH-CIC), \\ Faculty of Science, Mahidol University, Rama VI Road, Bangkok 10400, Thailand \\ E-mail: darunee.soo@mahidol.ac.th
}

\begin{abstract}
The diastereoselective reaction of the vicinal dianion, generated from di-(-)-menthyl succinate, with electrophiles, i.e. symmetrical ketones, aldehydes, and benzyl bromide was examined. The reactions with ketones and benzyl bromide gave products in good yields, however, with poor diastereoselectivities. The reaction with aldehydes in the presence of $\mathrm{ZnCl}_{2}$ preferably gave the anti-aldol adducts, which subsequently led to cis-paraconic esters again with moderate diastereoselectivity.
\end{abstract}

Keywords: Chiral succinic acid derivatives, di-(-)-menthyl succinate, vicinal dianion, paraconic esters

\section{Introduction}

Considerable attention has been focused on the synthetic utilities of succinic acid derivatives as they can serve as four-carbon building blocks in organic synthesis. ${ }^{1}$ Vicinal dianions generated from achiral succinic acid derivatives were found to be versatile synthetic intermediates for the synthesis of various types of compounds, ${ }^{2}$ such as mono- or dialkylated 1,4-dicarboxylic acid derivatives and ring-annulation products. Additionally, condensation of the vicinal dianion of diethyl succinate with aldehydes and ketones provided a straightforward route to paraconic esters. $^{2 \mathrm{p}}$ Paraconic acids are a family of $\gamma$-butyrolactones which possess important biological activities, such as antitumor, antifungal, and antibacterial activities. ${ }^{3}$ In connection with our previous results and research interest ${ }^{4}$ aimed at asymmetric synthetic strategies to chiral paraconic ester and chiral $\gamma$-butyrolactone frameworks, especially those in some bioactive lignan natural products, ${ }^{5}$ we became interested in employing the vicinal dianions, derived from the known chiral succinic acid derivatives. ${ }^{6}$ Diastereoselective reaction of the vicinal dianion derived from di-(-)-menthyl succinate (1) was focused in this study. The synthetic utility of $\mathbf{1}$ in 
asymmetric reactions was first disclosed by Yamamoto. ${ }^{7}$ Additionally, the dianion 2 generated from 1 was reported to react with 1, $\omega$-dihalides and ditosylates with high stereoselectivity leading to several chiral carbocyclic frameworks. ${ }^{8,9}$ Based on these precedent works, we therefore anticipated that the dianion $\mathbf{2}$ would react diastereoselectively with simple alkyl halides and carbonyl compounds leading to chiral $\gamma$-butyrolactone and chiral paraconic ester frameworks (Scheme 1). Herein, we wish to report the results of our study.

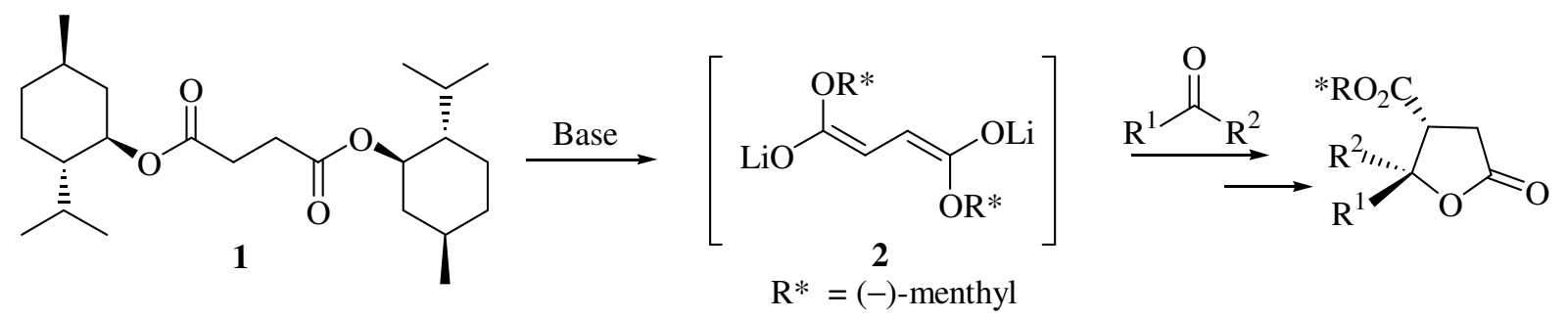

\section{Scheme 1}

\section{Results and Discussion}

Treatment of $\mathbf{1}$, readily prepared from succinic anhydride and (-)-menthol in the presence of a catalytic amount of $p$-TsOH, ${ }^{9}$ with two equivalents of lithium 2,2,6,6-tetramethylpiperidide (LTMP) in THF at $-78{ }^{\circ} \mathrm{C}$ for $1 \mathrm{~h}$ provided the dianion 2 (Scheme 2). Initially, the $\pi$-facial diastereoselection of the reaction of $\mathbf{2}$ with electrophiles was investigated by reacting $\mathbf{2}$ with a symmetrical ketone, cyclohexanone ( 1 equiv.), at $-78{ }^{\circ} \mathrm{C}$ for $1 \mathrm{~h}$. In the preliminary experiment, when the reaction mixture was quenched by using a saturated aqueous solution of $\mathrm{NH}_{4} \mathrm{Cl}$ at -78 ${ }^{\circ} \mathrm{C}$ followed by slowly warming up to room temperature, the adduct $\mathbf{3 a}$ and paraconic ester $\mathbf{4 a}$ were obtained in $54 \%$ and $18 \%$ yields, respectively, after chromatographic purification. This result implied that the lactonization of $\mathbf{3 a}$ to $\mathbf{4 a}$ readily took place under mildly acidic work-up conditions. The adduct $\mathbf{3 a}$ can be quantitatively converted into the corresponding paraconic ester 4a by treatment with a catalytic amount of $p-\mathrm{TsOH}$ in $\mathrm{CH}_{2} \mathrm{Cl}_{2}$ at room temperature overnight. Therefore, we decided to consecutively perform the lactonization reaction of the crude mixture obtained from the addition reaction and determined the diastereoselectivity of the reaction from lactone $\mathbf{4 a}$. 


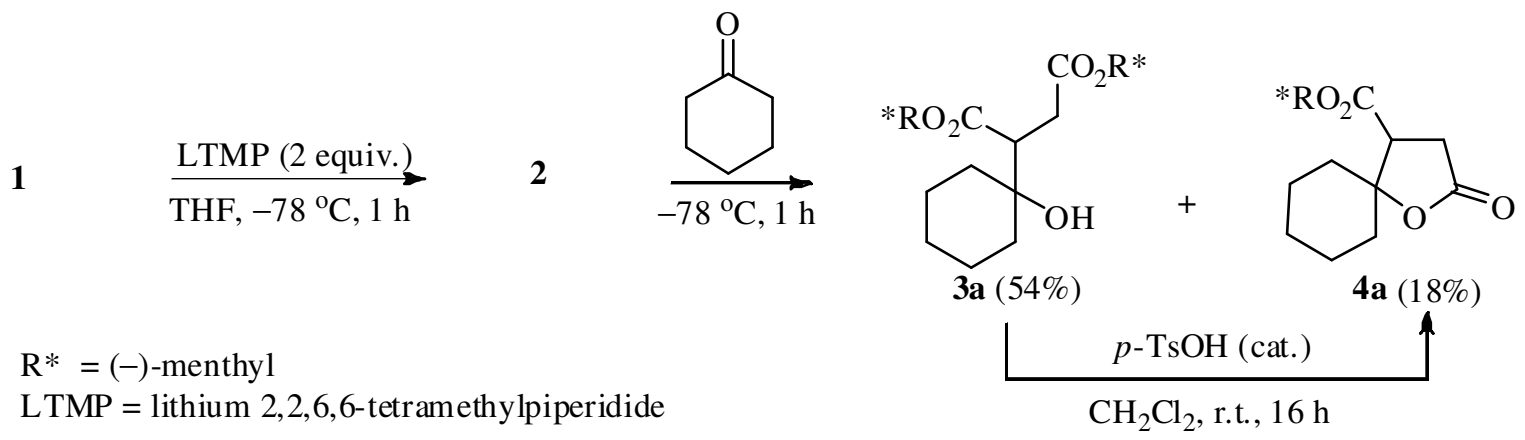

Scheme 2. Generation and reaction of the dianion 2 with cyclohexanone followed by lactonization.

The reaction of 2 with cyclohexanone ( 1 equiv.) at $-78{ }^{\circ} \mathrm{C}$ for $1 \mathrm{~h}$ followed by lactonization provided $4 \mathbf{a}$ in $69 \%$ yield as a 1:1.3 mixture of diastereomers as determined by using capillary GC (Table 1, entry 1). Attempts to separate the isomers in order to obtain a pure diastereomer by means of chromatographic technique were unsuccessful. Based on the previously reported works, ${ }^{7-9}$ the lack of diastereoselection in our study was surprising. The reactions of 2 with other symmetrical ketones including cyclopentanone, cycloheptanone, acetone, and 3-pentanone were also evaluated. The respective paraconic esters $\mathbf{4 b} \mathbf{b} \mathbf{4} \mathbf{e}$ were obtained in good yields as mixtures of diastereomers, each of which was unable to be separated by chromatography (Table 1, entries $2-5)$.

Table 1. Reaction of the dianion 2 with symmetrical ketones

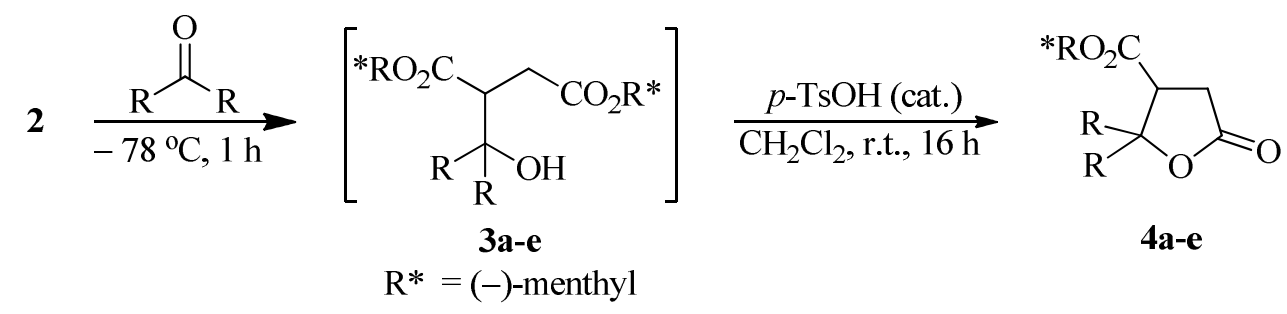

\begin{tabular}{llcc}
\hline Entry & Paraconic Ester $\mathbf{4}$ & ${\text { Yield }(\%)^{\mathrm{a}}}$ & Diastereomeric Ratio $^{\mathrm{b}}$ \\
\hline
\end{tabular}


Table 1. Continued

\begin{tabular}{cccc}
\hline Entry & Paraconic Ester 4 & ${\text { Yield }(\%)^{\mathrm{a}}}^{\text {Diastereomeric Ratio }^{\mathrm{b}}}$ \\
\hline & & & \\
\end{tabular}

${ }^{\mathrm{a}}$ Yields of isolated products. All compounds were fully characterized by ${ }^{1} \mathrm{H}-$ and ${ }^{13} \mathrm{C}-\mathrm{NMR}, \mathrm{MS}$, and $\mathrm{CHN}$ analyses.

${ }^{\mathrm{b}}$ Determined by capillary GC (see Experimental Part).

Asymmetric induction of $\mathbf{2}$ in the aldol reaction with aldehydes was next investigated. Cinnamaldehyde was chosen as a substrate since it was anticipated that the corresponding chiral paraconic ester 5a could be converted into various enantiopure paraconic acids via cross olefin metathesis with an appropriate alkene, followed by reduction of the resulting double bond. Under the standard reaction conditions used for ketones, the reaction of $\mathbf{2}$ with cinnamaldehyde (1 equiv.) provided cis-5a, trans-5a, and product 6, after chromatography, in 32\%, 25\%, and 15\% yields, respectively (Scheme 3, Table 2, entry 1). Compound 6 was obtained as a single diastereomer, whereas cis-5a and trans-5a were obtained as an inseparable 1:1 mixtures of diastereomers (cis-5aA+cis-5aB and trans-5aA+trans-5aB). Similar results were obtained when 2 was allowed to react with cinnamaldehyde (1 equiv.) in the presence of $\mathrm{ZnCl}_{2}$ (1 equiv.). Cis5a, trans-5a, and compound $\mathbf{6}$ were isolated in 37\%, 22\%, and 10\% yields, respectively (Table 2, entry 2). Interestingly, when two equivalents of cinnamaldehyde were employed to react with 2 at $-78{ }^{\circ} \mathrm{C}$ for $3.5 \mathrm{~h}$, compound 6 was isolated in $56 \%$ yield as a sole product (Table 2, entry 3 ). When two equivalents of $\mathrm{ZnCl}_{2}$ were employed, the reaction proceeded with higher selectivity favoring the anti aldol adduct leading to cis-5a as a major product (52\%) together with trans-5a (24\%), and compound 6 was not observed (Table 2, entry 4). The relative stereoselectivity of the aldol reaction leading to cis-5a as a major product was assumed based on the literature. ${ }^{10}$ The relative stereochemistry of cis- and trans-5a was further confirmed upon hydrolysis to the corresponding paraconic acids ${ }^{11}$ and the comparison of their ${ }^{1} \mathrm{H}-\mathrm{NMR}$ data with those reported in the literature. ${ }^{1 \mathrm{f}}$ Although the ratio between cis- and trans-5a could be improved and both diastereomers could easily be separated by chromatography, poor $\pi$-facial selection of the 
dianion 2 towards cinnamaldehyde led to an inseparable 1:1 mixture of cis-5aA and cis-5aB as well as a 1:1 mixture of trans-5aA and trans-5aB.

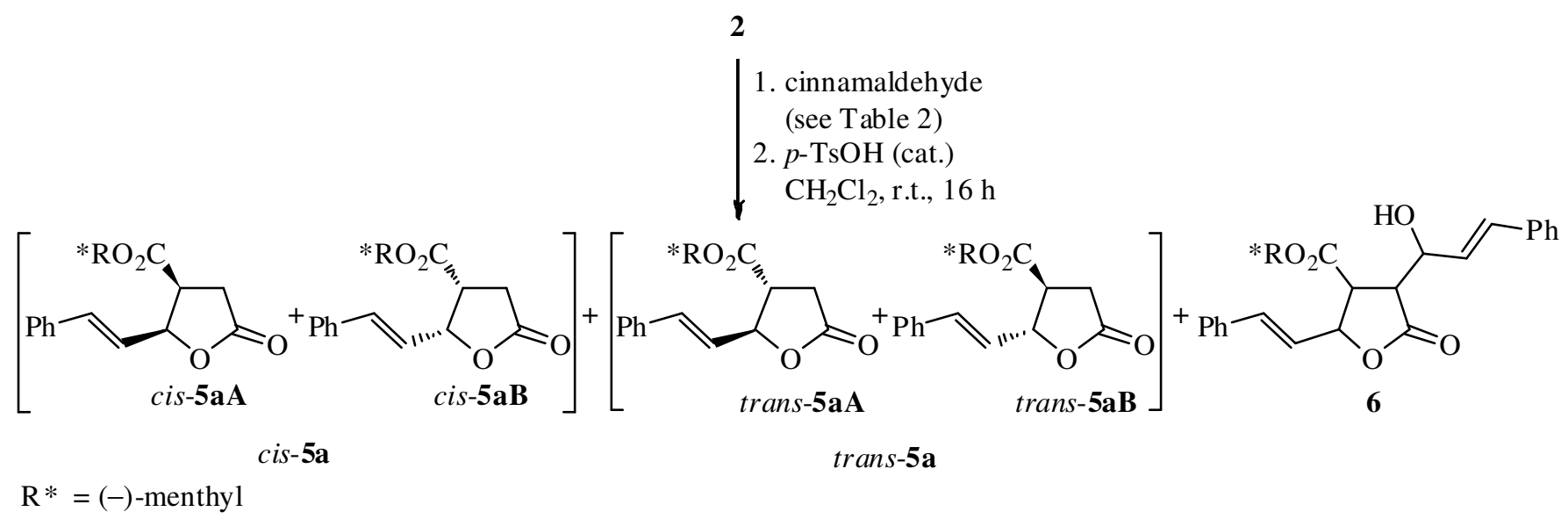

Scheme 3. Reaction of the dianion 2 with cinnamaldehyde.

Table 2. Optimization for the reaction of the dianion 2 with cinnamaldehyde

\begin{tabular}{ccccccc}
\hline Entry & $\begin{array}{c}\mathrm{ZnCl}_{2} \\
\text { (equiv.) }\end{array}$ & Conditions & $\begin{array}{c}\text { Cinnamaldehyde } \\
\text { (equiv.) }\end{array}$ & $\begin{array}{c}\text { cis-5a } \\
(\%)^{\mathrm{a}}\end{array}$ & $\begin{array}{c}\text { trans-5a } \\
(\%)^{\mathrm{a}}\end{array}$ & $\begin{array}{c}\mathbf{6} \\
(\%)^{\mathrm{a}}\end{array}$ \\
\hline 1 & - & $-78{ }^{\circ} \mathrm{C}, 1 \mathrm{~h}$ & 1 & 32 & 25 & 15 \\
2 & 1 & $-78{ }^{\circ} \mathrm{C}, 1 \mathrm{~h}$ & 1 & 37 & 22 & 10 \\
3 & - & $-78{ }^{\circ} \mathrm{C}, 3.5 \mathrm{~h}$ & 2 & - & - & 56 \\
4 & 2 & $-78{ }^{\circ} \mathrm{C}$ to r.t., $16 \mathrm{~h}$ & 1 & 52 & 24 & - \\
\hline
\end{tabular}

${ }^{\mathrm{a}}$ Yields of isolated products. All compounds were fully characterized by ${ }^{1} \mathrm{H}$ - and ${ }^{13} \mathrm{C}-\mathrm{NMR}, \mathrm{MS}$, and HRMS.

Similar results were observed, when 2 was allowed to react with 3,4,5trimethoxybenzaldehyde (1 equiv.) in the presence of $\mathrm{ZnCl}_{2}$ (2 equiv.). Cis-5b and trans-5b, each as a mixture of cis-5bA+cis-5bB and trans-5bA+trans-5bB, respectively, were isolated in $59 \%$ and $12 \%$ yields (Scheme 4). In contrast to the result indicated in Table 2, entry 3, the reaction of 2 with two equivalents of 3,4,5-trimethoxybenzaldehyde under similar reaction conditions did not give the expected product 7. Instead, a mixture of products including cis-5b and trans-5b was obtained. 


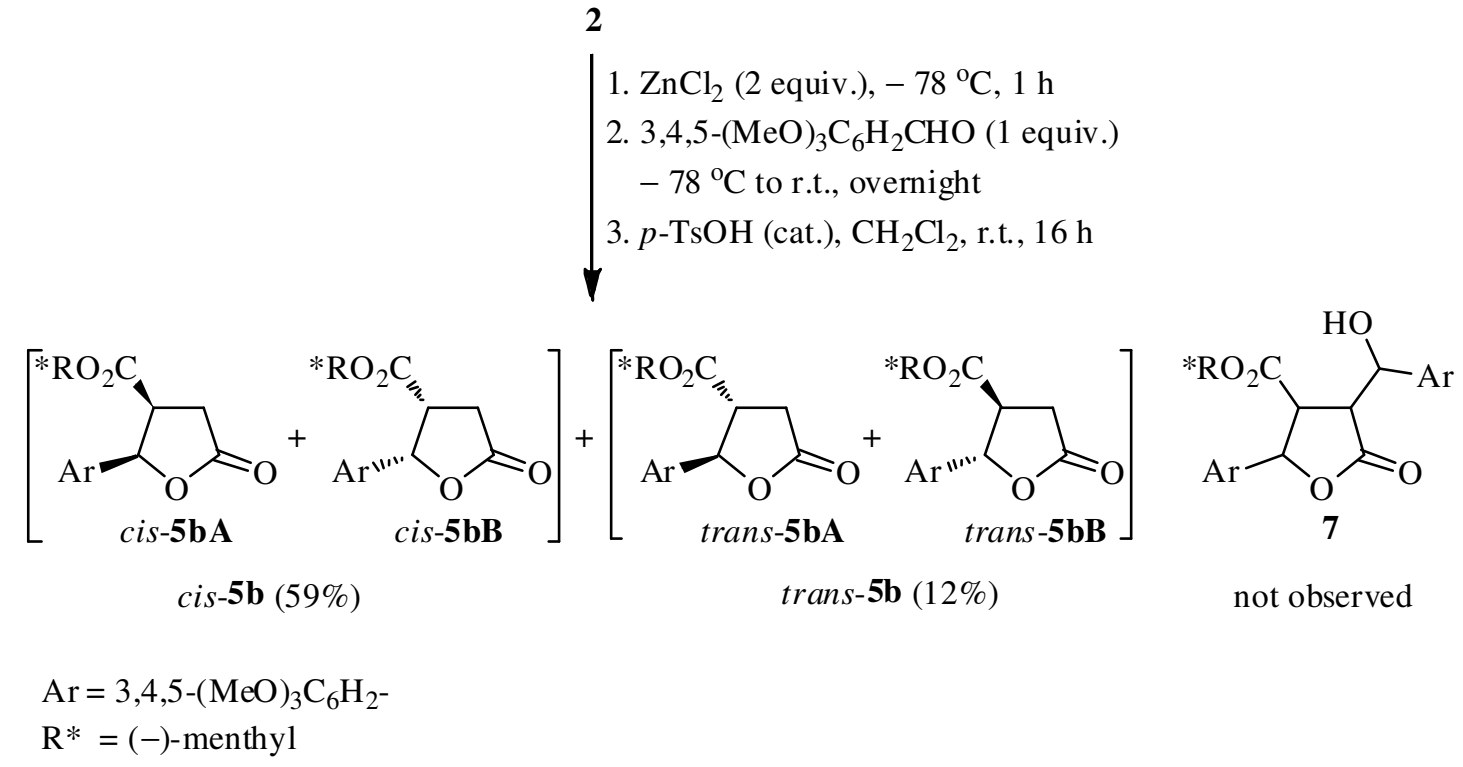

Scheme 4. Reaction of the dianion 2 with 3,4,5-trimethoxybenzaldehyde.

Finally, the diastereoselectivity in alkylation reactions of $\mathbf{2}$ was also studied (Scheme 5). The reaction of 2 with benzyl bromide ( 1 equiv.) at $-78{ }^{\circ} \mathrm{C}$ for $2 \mathrm{~h}$ afforded monobenzylated product $\mathbf{8}$ in $89 \%$ yield as an inseparable mixture of diastereoisomers $(\mathrm{dr}=1: 1.2) .{ }^{12}$ Similar results were also observed when 2 was treated with two equivalents of benzyl bromide at $-78{ }^{\circ} \mathrm{C}$ and the reaction was allowed to warm up to room temperature overnight $(16 \mathrm{~h})$; monobenzylated product 8 and dibenzylated product 9 were obtained in $44 \%(\mathrm{dr}=1: 1)$ and $7 \%$ yields, respectively.

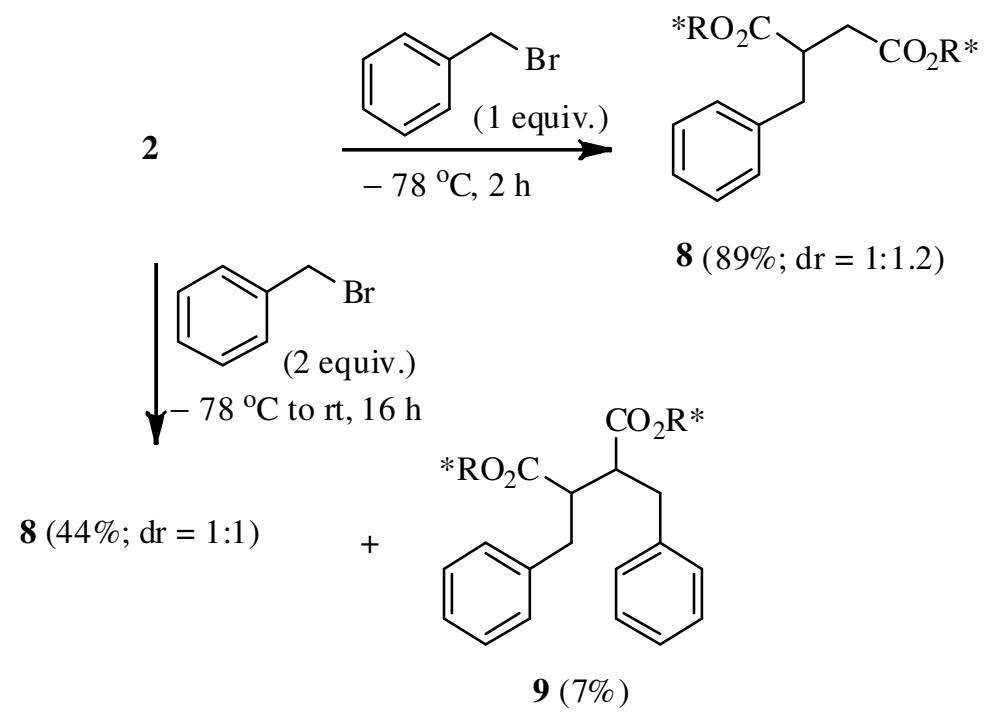

Scheme 5. Reaction of the dianion 2 with benzyl bromide. 
At this point, the observed stereochemical outcomes should be discussed. The major conformation of $\mathbf{2}$ was proved to be $S$-trans-E,E-enolate (Figure 1) when LTMP was used as a base in THF at $-78{ }^{\circ} \mathrm{C} .{ }^{7}$ Considering the major geometry of 2 , the approach of electrophiles from the less hindered $\mathrm{Si}$-face leading to the $(R)$-products should be expected to proceed with high diastereoselection. However, it was evident that $\mathbf{2}$ allowed the approach of electrophiles from both $R e$ - and $\mathrm{Si}$-faces since the mixture of diastereomers was observed in all cases.

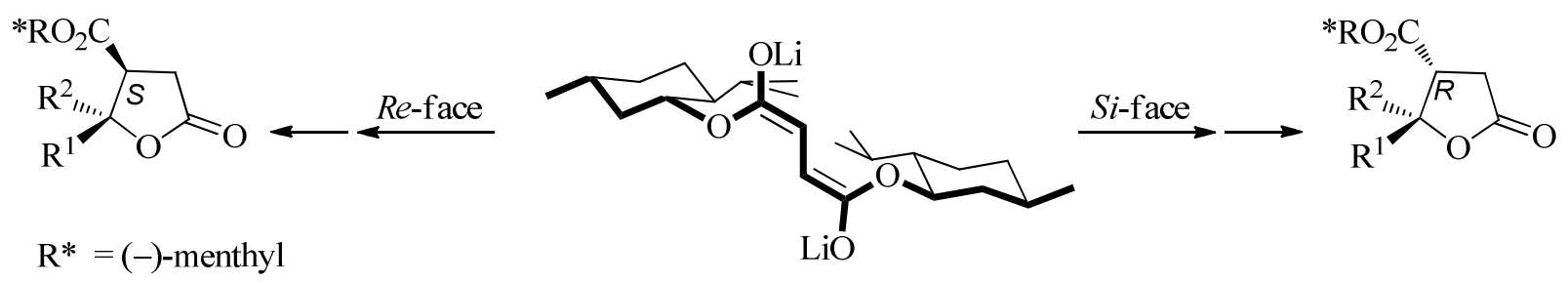

Figure 1. $S$-trans-(E,E)-Enolate.

\section{Conclusions}

In conclusion, the detailed investigation on the diastereoselective reaction of the vicinal dianion 2, generated from di-(-)-menthyl succinate (1), with electrophiles, i.e. symmetrical ketones, aldehydes, and benzyl bromide was evaluated. In contrast to those observed when 1, $\omega$-dihalides and ditosylates were employed as electrophiles, the reaction of $\mathbf{2}$ with simple ketones and benzyl bromide proceeded to give the products in good yields, however, with poor diastereoselectivities. Similarly, the reaction of $\mathbf{2}$ with aldehydes in the presence of $\mathrm{ZnCl}_{2}$ afforded predominately the anti-aldol adducts which led to the cis-paraconic esters again with moderate diastereoselectivity. The results described herein may be useful for organic chemists studying in this area.

\section{Experimental Section}

General. Melting points (uncorrected) were measured on a Büchi 501 apparatus. Capillary GLC analyses were performed on HP 6890 Series equipped with flame ionization detector; chromatographic column was $25 \mathrm{~m}$ x $0.32 \mathrm{~mm}$ x $0.52 \mu \mathrm{m}$, HP-5, 5\% diphenyl and $95 \%$ dimethylpolysiloxane. All analyses were carried out using flow rate $1 \mathrm{~mL} / \mathrm{min}$, split ratio $25, \mathrm{H}_{2}-$ flow $30 \mathrm{~mL} / \mathrm{min}$, Air-flow $400 \mathrm{~mL} / \mathrm{min}, \mathrm{N}_{2}$-flow $28.5 \mathrm{~mL} / \mathrm{min}$, combined flow $30 \mathrm{~mL} / \mathrm{min}$; peak areas and retention times were recorded using a HP 3369 Series III Integrator. Optical rotation was measured on a Jasco P-1020 polarimeter. IR spectra were recorded on GX FT-IR system Perkin Elmer infrared spectrometer. ${ }^{1} \mathrm{H}$ and ${ }^{13} \mathrm{C}$ NMR spectra were recorded on either Bruker DPX-300 or Bruker-500 spectrometer. The mass spectra were recorded by using ThermoFinnigan Polaris Q mass spectrometer. Elemental analyses were performed with a Perkin 
Elmer Elemental Analyzer $2400 \mathrm{CHN}$. The high resolution mass spectra were recorded on either HR-TOF-MS Micromass model VQ-TOF2 or Finnigan MAT 95 mass spectrometer. All reactions were carried out with magnetic stirring and in oven-dried glassware under an Ar atmosphere. THF was distilled from sodium-benzophenone ketyl. The molarity of $n$-BuLi (in hexane) was determined by titration with diphenylacetic acid in $\mathrm{THF}$ at $0{ }^{\circ} \mathrm{C}$. 2,2,6,6Tetramethylpiperidine was distilled over $\mathrm{CaH}_{2}$. Column chromatography (CC) was performed by using Merck silica gel 60H (Art. 7736). Preparative thin-layer chromatography (PLC) was performed by using Merck silica gel $60\left(\mathrm{PF}_{254}\right.$, Art. 7747). Di-(-)-menthyl succinate (1) was synthesized from succinic anhydride and (-)-menthol according to the lituratue. ${ }^{9}$

\section{General procedure for the synthesis of 4}

A solution of 1 ( $2 \mathrm{mmol})$ in dry THF $(2 \mathrm{~mL})$ was added dropwise to a THF solution of lithium 2,2,6,6-tetramethylpiperidide (LTMP) [prepared by reacting 2,2,6,6-tetramethylpiperidine $(0.75$ $\mathrm{mL}, 4.4 \mathrm{mmol})$ in THF (6 mL) with $n$-BuLi $(1.49 \mathrm{M}$ in hexane, $2.8 \mathrm{~mL}, 4.2 \mathrm{mmol})$ at $-78{ }^{\circ} \mathrm{C}$ for $1 \mathrm{~h}]$ at $-78{ }^{\circ} \mathrm{C}$ under an $\mathrm{Ar}$ atmosphere. After stirring at $-78{ }^{\circ} \mathrm{C}$ for $1 \mathrm{~h}$, the resulting solution of 2 was treated with a solution of ketone $(2 \mathrm{mmol})$ in THF $(2 \mathrm{~mL})$. The mixture was stirred at $-78^{\circ} \mathrm{C}$ for $1 \mathrm{~h}$, then quenched with $2 \mathrm{M} \mathrm{HCl}(5 \mathrm{~mL}, \mathrm{pH} 1-2)$, warmed to r.t., diluted with $\mathrm{H}_{2} \mathrm{O}(20 \mathrm{~mL})$, and extracted with EtOAc $(4 \times 25 \mathrm{~mL})$. The combined extracts were washed with $\mathrm{H}_{2} \mathrm{O}$, brine and dried (anh. $\mathrm{Na}_{2} \mathrm{SO}_{4}$ ). After removal of solvents, the crude product was treated with $p$-TsOH (13 $\mathrm{mg}$ ) in dry $\mathrm{CH}_{2} \mathrm{Cl}_{2}(5 \mathrm{~mL})$. After stirring at r.t. for $16 \mathrm{~h}$, the mixture was quenched with $\mathrm{H}_{2} \mathrm{O}$ and extracted with EtOAc $(4 \times 25 \mathrm{~mL})$. The combined extracts were washed with $\mathrm{H}_{2} \mathrm{O}$, brine and dried (anh. $\mathrm{Na}_{2} \mathrm{SO}_{4}$ ). The crude product was purified by column chromatography.

(2-Isopropyl-5-methylcyclohexyl)-2-oxo-1-oxaspiro[4.5]decane-4-carboxylate (4a). White solid, yield 69\%, $464 \mathrm{mg}$, $\mathrm{mp} 70-74{ }^{\circ} \mathrm{C}\left(\mathrm{CH}_{2} \mathrm{Cl}_{2} /\right.$ hexanes $), \mathrm{dr}=1: 1.3\left(\mathrm{t}_{\mathrm{R} 1}=11.139, \mathrm{t}_{\mathrm{R} 2}=11.487\right.$, injector temp. $260{ }^{\circ} \mathrm{C}$, detector temp. $270{ }^{\circ} \mathrm{C}$, oven temp. $240{ }^{\circ} \mathrm{C}$ ); IR (nujol): $v 1791 s, 1733 s$, $1418 \mathrm{~m} \mathrm{~cm}^{-1} .{ }^{1} \mathrm{H}-\mathrm{NMR}\left(300 \mathrm{MHz}, \mathrm{CDCl}_{3}\right.$, determined for two isomers): $\delta 4.74$ (dt, $J 4.4,10.8$, $1 \mathrm{H}, \mathrm{CH}), 4.70(\mathrm{dt}, J$ 4.4, 10.8, $1 \mathrm{H}, \mathrm{CH}), 3.12-2.98(\mathrm{~m}, 4 \mathrm{H}, 2 \times \mathrm{CHCH}), 2.75-2.60(\mathrm{~m}, 2 \mathrm{H}$, $2 \times \mathrm{CH} H$ ), 2.10-1.58, 1.58-0.80 and 0.80-0.70 (each $\mathrm{m}, 56 \mathrm{H}$, methine, methylene and methyl protons). ${ }^{13} \mathrm{C}$-NMR $\left(75 \mathrm{MHz}, \mathrm{CDCl}_{3}\right.$, determined for two isomers): $\delta 174.4(2 \times \mathrm{CO}), 169.5(\mathrm{CO})$ $169.4(\mathrm{CO}), 86.0(\mathrm{C}), 85.9(\mathrm{C}), 76.0(\mathrm{CH}), 75.7(\mathrm{CH}), 51.1(\mathrm{CH}), 50.5(\mathrm{CH}), 46.9(\mathrm{CH}), 46.7$ $(\mathrm{CH}), 40.7\left(\mathrm{CH}_{2}\right), 40.6\left(\mathrm{CH}_{2}\right), 37.31\left(\mathrm{CH}_{2}\right), 37.27\left(\mathrm{CH}_{2}\right), 34.04\left(\mathrm{CH}_{2}\right), 34.0\left(\mathrm{CH}_{2}\right), 32.20\left(\mathrm{CH}_{2}\right)$, $32.19\left(\mathrm{CH}_{2}\right), 31.7\left(\mathrm{CH}_{2}\right), 31.5\left(\mathrm{CH}_{2}\right), 31.3(2 \times \mathrm{CH}), 26.2(\mathrm{CH}), 26.1(\mathrm{CH}), 24.93\left(\mathrm{CH}_{2}\right), 24.91$ $\left(\mathrm{CH}_{2}\right), 23.2\left(\mathrm{CH}_{2}\right), 22.7\left(\mathrm{CH}_{2}\right), 22.5\left(\mathrm{CH}_{2}\right), 22.4\left(\mathrm{CH}_{2}\right), 22.0\left(\mathrm{CH}_{3}\right), 21.9\left(\mathrm{CH}_{3}\right), 21.6\left(2 \times \mathrm{CH}_{2}\right)$, $20.8\left(\mathrm{CH}_{3}\right), 20.7\left(\mathrm{CH}_{3}\right), 16.1\left(\mathrm{CH}_{3}\right), 15.6\left(\mathrm{CH}_{3}\right) . \mathrm{MS}, \mathrm{m} / z,(\%)=336\left(\mathrm{M}^{+}\right)$; Anal. Calcd. for $\mathrm{C}_{20} \mathrm{H}_{32} \mathrm{O}_{4}$ (336.23): C, 71.41; H, 9.58\%. Found: C, 70.98; H, 9.27\%.

(2-Isopropyl-5-methylcyclohexyl)-2-oxo-1-oxaspiro[4.4]nonane-4-carboxylate

(4b). Colorless viscous oil, yield $79 \%, 508 \mathrm{mg}, \mathrm{dr}=1: 1.4\left(\mathrm{t}_{\mathrm{R} 1}=19.363, \mathrm{t}_{\mathrm{R} 2}=19.867\right.$, injector temp. $230{ }^{\circ} \mathrm{C}$, detector temp. $250{ }^{\circ} \mathrm{C}$, oven temp. $220{ }^{\circ} \mathrm{C}$ ); IR (neat): $v 2957 s, 2872 s, 1785 s, 1731 s$, $1238 s, 1166 \mathrm{sm}^{-1}$. ${ }^{1} \mathrm{H}-\mathrm{NMR}\left(300 \mathrm{MHz}, \mathrm{CDCl}_{3}\right.$, determined for two isomers): $\delta$ 4.80-4.65 (m, $2 \mathrm{H}, 2 \times \mathrm{CH}), 3.38-3.28(\mathrm{~m}, 2 \mathrm{H}, 2 \times \mathrm{CH}), 2.98(\mathrm{dd}, J 8.4,17.6,2 \mathrm{H}, 2 \times \mathrm{CHH}), 2.72(\mathrm{dd}, J 8.4,17.6$, 
1H, CHH), 2.71 (dd, $J$ 8.4, 17.6, 1H, CHH), 2.12-1.59, 1.59-1.33, 1.16-0.80 and 0.80-0.69 (each $\mathrm{m}, 52 \mathrm{H}$, methine, methylene and methyl protons). ${ }^{13} \mathrm{C}-\mathrm{NMR}\left(75 \mathrm{MHz}, \mathrm{CDCl}_{3}\right.$, determined for two isomers): $\delta 174.2(\mathrm{CO}), 174.1(\mathrm{CO}), 169.7(\mathrm{CO}), 169.6(\mathrm{CO}), 94.8(\mathrm{C}), 94.7(\mathrm{C}), 75.9(\mathrm{CH})$, $75.7(\mathrm{CH}), 48.5(\mathrm{CH}), 48.0(\mathrm{CH}), 46.8(\mathrm{CH}), 46.7(\mathrm{CH}), 40.7\left(\mathrm{CH}_{2}\right), 40.6\left(\mathrm{CH}_{2}\right), 38.8\left(\mathrm{CH}_{2}\right)$, $38.7\left(\mathrm{CH}_{2}\right), 34.4\left(\mathrm{CH}_{2}\right), 34.2\left(\mathrm{CH}_{2}\right), 34.0\left(\mathrm{CH}_{2}\right), 33.9\left(\mathrm{CH}_{2}\right), 32.9\left(\mathrm{CH}_{2}\right), 32.6\left(\mathrm{CH}_{2}\right), 31.3$ $(2 \times \mathrm{CH}), 26.2(\mathrm{CH}), 26.0(\mathrm{CH}), 23.9\left(\mathrm{CH}_{2}\right), 23.7\left(\mathrm{CH}_{2}\right), 23.3\left(\mathrm{CH}_{2}\right), 23.2\left(\mathrm{CH}_{2}\right), 23.1\left(\mathrm{CH}_{2}\right), 22.8$ $\left(\mathrm{CH}_{2}\right), 21.8\left(\mathrm{CH}_{3}\right), 21.9\left(\mathrm{CH}_{3}\right), 20.7\left(\mathrm{CH}_{3}\right), 20.6\left(\mathrm{CH}_{3}\right), 16.1\left(\mathrm{CH}_{3}\right), 15.6\left(\mathrm{CH}_{3}\right) . \mathrm{MS}, \mathrm{m} / z,(\%)=$ $322\left(\mathrm{M}^{+}\right)$. Anal. Calcd. for $\mathrm{C}_{19} \mathrm{H}_{30} \mathrm{O}_{4}$ (322.21): C, 70.78; H, 9.38\%. Found: C, 70.60; H, 9.38\%.

(2-Isopropyl-5-methylcyclohexyl)-2-oxo-1-oxaspiro[4.6]undecane-4-carboxylate

(4c). Colorless viscous oil, yield $81 \%, 571 \mathrm{mg}, \mathrm{dr}=1: 1.1\left(\mathrm{t}_{\mathrm{R} 1}=11.395, \mathrm{t}_{\mathrm{R} 2}=11.397\right.$, injector temp. $230{ }^{\circ} \mathrm{C}$, detector temp. $250{ }^{\circ} \mathrm{C}$, oven temp. $220{ }^{\circ} \mathrm{C}$ ); IR (neat): $v 2930 s, 2869 s, 1784 s, 1732 s$, $1485 s, 1372 s, 1246 s, 1197 s, 1173 s \mathrm{~cm}^{-1}$. ${ }^{1} \mathrm{H}-\mathrm{NMR}\left(300 \mathrm{MHz}, \mathrm{CDCl}_{3}\right.$, determined for two isomers): $\delta$ 4.80-4.63 $(\mathrm{m}, 2 \mathrm{H}, 2 \times \mathrm{CH}), 3.17-2.93(\mathrm{~m}, 4 \mathrm{H}, 2 \times \mathrm{CHCHH}), 2.72-2.59(\mathrm{~m}, 2 \mathrm{H}$, $2 \times \mathrm{CH} H$ ), 2.15-1.35 and 1.20-0.70 (each $\mathrm{m}, 60 \mathrm{H}$, methine, methylene and methyl protons). ${ }^{13} \mathrm{C}-$ NMR (75 MHz, $\mathrm{CDCl}_{3}$, determined for two isomers): $\delta 174.3(2 \times \mathrm{CO}), 169.5(\mathrm{CO}), 169.4(\mathrm{CO})$, $89.6(2 \times \mathrm{C}), 76.0(\mathrm{CH}), 75.7(\mathrm{CH}), 51.6(\mathrm{CH}), 51.5(\mathrm{CH}), 46.9(\mathrm{CH}), 46.7(\mathrm{CH}), 41.7\left(\mathrm{CH}_{2}\right), 41.6$ $\left(\mathrm{CH}_{2}\right), 40.7\left(\mathrm{CH}_{2}\right), 40.6\left(\mathrm{CH}_{2}\right), 34.4\left(\mathrm{CH}_{2}\right), 34.2\left(\mathrm{CH}_{2}\right), 34.1\left(\mathrm{CH}_{2}\right), 34.0\left(\mathrm{CH}_{2}\right), 31.9\left(\mathrm{CH}_{2}\right), 31.8$ $\left(\mathrm{CH}_{2}\right), 31.3(\mathrm{CH}), 31.2(\mathrm{CH}), 28.9\left(\mathrm{CH}_{2}\right), 28.8\left(\mathrm{CH}_{2}\right), 28.3\left(\mathrm{CH}_{2}\right), 28.2\left(\mathrm{CH}_{2}\right), 26.2(\mathrm{CH}), 26.0$ $(\mathrm{CH}), 23.2\left(\mathrm{CH}_{2}\right), 22.8\left(\mathrm{CH}_{2}\right), 22.6\left(\mathrm{CH}_{2}\right), 22.5\left(\mathrm{CH}_{2}\right), 21.9\left(\mathrm{CH}_{3}\right), 21.8\left(\mathrm{CH}_{3}\right), 21.6\left(\mathrm{CH}_{2}\right), 21.4$ $\left(\mathrm{CH}_{2}\right), 20.8\left(\mathrm{CH}_{3}\right), 20.7\left(\mathrm{CH}_{3}\right), 16.1\left(\mathrm{CH}_{3}\right), 15.7\left(\mathrm{CH}_{3}\right) . \mathrm{MS}, \mathrm{m} / z,(\%)=351(\mathrm{M}+1)^{+}$. Anal. Calcd. for $\mathrm{C}_{21} \mathrm{H}_{34} \mathrm{O}_{4}$ (350.25): C, 71.96; $\mathrm{H} 9.78 \%$. Found: C, 72.12; H, 9.39\%.

(2-Isopropyl-5-methylcyclohexyl)-2,2-dimethyl-5-oxotetrahydrofuran-3-carboxylate (4d). Colorless viscous oil, yield 76\%, $448 \mathrm{mg}, \mathrm{dr}=1: 1\left(\mathrm{t}_{\mathrm{R} 1}=37.263, \mathrm{t}_{\mathrm{R} 2}=38.129\right.$, injector temp. 200 ${ }^{\circ} \mathrm{C}$, detector temp. $250{ }^{\circ} \mathrm{C}$, oven temp. $175{ }^{\circ} \mathrm{C}$ ). IR (neat): $v 2957 s, 2872 s, 1785 s, 1731 s, 1270 s$, $1253 s, 1212 s, 1175 s, 1120 s \mathrm{~cm}^{-1}$. ${ }^{1} \mathrm{H}-\mathrm{NMR}\left(300 \mathrm{MHz}, \mathrm{CDCl}_{3}\right.$, determined for two isomers): $\delta$ $4.69(\mathrm{dt}, J 4.4,10.7,1 \mathrm{H}, \mathrm{CH}), 4.65(\mathrm{dt}, J 4.4,10.7,1 \mathrm{H}, \mathrm{CH}), 3.16-2.93(\mathrm{~m}, 4 \mathrm{H}, 2 \times \mathrm{CHCHH})$, $2.63(\mathrm{dd}, J$ 8.0, 17.2, 1H, CHH), 2.62 (dd, $J$ 8.0, 17.2, 1H, CHH), 2.00-1.86, 1.86-1.69, 1.69$1.57,1.57-1.50,1.50-1.29,1.29-1.22,1.09-0.72$ and 0.72-0.63 (each $\mathrm{m}, 48 \mathrm{H}$, methine, methylene and methyl protons). ${ }^{13} \mathrm{C}-\mathrm{NMR}\left(75 \mathrm{MHz}, \mathrm{CDCl}_{3}\right.$, determined for two isomers): $\delta 174.0(\mathrm{CO})$, $173.9(\mathrm{CO}), 169.3(\mathrm{CO}), 169.2(\mathrm{CO}), 84.4(\mathrm{C}), 84.3(\mathrm{C}), 76.0(\mathrm{CH}), 75.7(\mathrm{CH}), 50.9(\mathrm{CH}), 50.4$ $(\mathrm{CH}), 46.8(\mathrm{CH}), 46.6(\mathrm{CH}), 40.6\left(2 \times \mathrm{CH}_{2}\right), 34.0\left(\mathrm{CH}_{2}\right), 33.9\left(\mathrm{CH}_{2}\right), 32.0\left(\mathrm{CH}_{2}\right), 31.6\left(\mathrm{CH}_{2}\right), 31.3$ $(2 \times \mathrm{CH}), 28.4\left(2 \times \mathrm{CH}_{3}\right), 26.2(\mathrm{CH}), 26.1(\mathrm{CH}), 23.3\left(\mathrm{CH}_{2}\right), 23.2\left(\mathrm{CH}_{3}\right), 23.1\left(\mathrm{CH}_{3}\right), 22.8\left(\mathrm{CH}_{2}\right)$, $21.9\left(\mathrm{CH}_{3}\right), 21.8\left(\mathrm{CH}_{3}\right), 20.7\left(\mathrm{CH}_{3}\right), 20.6\left(\mathrm{CH}_{3}\right), 16.2\left(\mathrm{CH}_{3}\right), 15.6\left(\mathrm{CH}_{3}\right) . \mathrm{MS}, \mathrm{m} / z,(\%)=296$ $\left(\mathrm{M}^{+}\right)$. Anal. Calcd. for $\mathrm{C}_{17} \mathrm{H}_{28} \mathrm{O}_{4}$ (296.20): C, 68.89; H, 9.52\%. Found: C, 68.65; H, 9.08\%.

(2-Isopropyl-5-methylcyclohexyl)-2,2-diethyl-5-oxotetrahydrofuran-3-carboxylate

(4e). White solid, yield $80 \%, 521 \mathrm{mg}, \mathrm{mp} 78-85{ }^{\circ} \mathrm{C}$ (hexanes), $\mathrm{dr}=1: 1.6\left(\mathrm{t}_{\mathrm{R} 1}=29.348, \mathrm{t}_{\mathrm{R} 2}=29.764\right.$, injector temp. $210{ }^{\circ} \mathrm{C}$, detector temp. $250{ }^{\circ} \mathrm{C}$, oven temp. (temp. program) 190-200 ${ }^{\circ} \mathrm{C}$. IR (nujol): v 1788s, $1725 s, 1225 \mathrm{sm}^{-1}$. ${ }^{1} \mathrm{H}-\mathrm{NMR}$ (300 MHz, $\mathrm{CDCl}_{3}$, determined for two isomers): $\delta$ $4.74(\mathrm{dt}, J 4.4,10.7,1 \mathrm{H}, \mathrm{CH}), 4.71(\mathrm{dt}, J 4.4,10.7,1 \mathrm{H}, \mathrm{CH}), 3.30(\mathrm{t}, J 9.4,1 \mathrm{H}, \mathrm{CH}), 3.29(\mathrm{t}, J$ 9.4, 1H, CH), 3.14-2.99 (m, 2H, 2×CHH), $2.70(\mathrm{dd}, J$ 9.4, 18.2, 1H, CHH), 2.69 (dd, J 9.4, 18.2, 
$1 \mathrm{H}, \mathrm{CH} H$ ), 2.10-1.33, 1.18-0.80 and 0.80-0.70 (each $\mathrm{m}, 56 \mathrm{H}$, methine, methylene and methyl protons). ${ }^{13} \mathrm{C}-\mathrm{NMR}\left(75 \mathrm{MHz}, \mathrm{CDCl}_{3}\right.$, determined for two isomers): $\delta 174.5(2 \times \mathrm{CO}), 169.7$ (2×CO), $89.2(\mathrm{C}), 89.1(\mathrm{C}), 76.1(\mathrm{CH}), 75.7(\mathrm{CH}), 47.1(\mathrm{CH}), 46.9(\mathrm{CH}), 46.8(\mathrm{CH}), 46.7(\mathrm{CH})$, $40.6\left(\mathrm{CH}_{2}\right), 40.5\left(\mathrm{CH}_{2}\right), 34.1\left(\mathrm{CH}_{2}\right), 34.0\left(\mathrm{CH}_{2}\right), 32.4\left(\mathrm{CH}_{2}\right), 32.2\left(\mathrm{CH}_{2}\right), 31.3(2 \times \mathrm{CH}), 29.8$ $\left(\mathrm{CH}_{2}\right), 29.7\left(\mathrm{CH}_{2}\right), 27.7\left(\mathrm{CH}_{2}\right), 27.6\left(\mathrm{CH}_{2}\right), 26.4(\mathrm{CH}), 26.1(\mathrm{CH}), 23.4\left(\mathrm{CH}_{2}\right), 22.9\left(\mathrm{CH}_{2}\right), 22.0$ $\left(\mathrm{CH}_{3}\right), 21.9\left(\mathrm{CH}_{3}\right), 20.8\left(\mathrm{CH}_{3}\right), 20.7\left(\mathrm{CH}_{3}\right), 16.3\left(\mathrm{CH}_{3}\right), 15.7\left(\mathrm{CH}_{3}\right), 7.63\left(2 \times \mathrm{CH}_{3}\right), 7.59\left(2 \times \mathrm{CH}_{3}\right)$. MS, $m / z,(\%)=324\left(\mathrm{M}^{+}\right)$. Anal. Calcd. for $\mathrm{C}_{19} \mathrm{H}_{32} \mathrm{O}_{4}$ (324.23): C, 70.34; H, 9.94\%. Found: C, 70.67; H, 9.84\%.

(2-Isopropyl-5-methylcyclohexyl)-5-oxo-2-styryltetrahydrofuran-3-carboxylate

(5a). Dienolate 2 generated from 1 ( $1 \mathrm{mmol}$ ) was treated with a solution of $\mathrm{ZnCl}_{2}(1 \mathrm{M}$ in $\mathrm{THF}, 2$ mmol) and stirred at $-78{ }^{\circ} \mathrm{C}$ for $1 \mathrm{~h}$. A solution of cinnamaldehyde (132 mg, $\left.1 \mathrm{mmol}\right)$ in THF (2 $\mathrm{mL}$ ) was added dropwise at $-78{ }^{\circ} \mathrm{C}$. The mixture was allowed to stir and slowly warmed up to r.t. overnight, quenched with $2 \mathrm{M} \mathrm{HCl}(5 \mathrm{~mL}, \mathrm{pH} 1-2)$, diluted with $\mathrm{H}_{2} \mathrm{O}$ (20 mL) and extracted with EtOAc $(4 \times 25 \mathrm{~mL})$. The combined extracts were washed with $\mathrm{H}_{2} \mathrm{O}$, brine and dried (anh. $\left.\mathrm{Na}_{2} \mathrm{SO}_{4}\right)$. After removal of solvents, the crude product was treated with $p$-TsOH $(13 \mathrm{mg})$ in dry $\mathrm{CH}_{2} \mathrm{Cl}_{2}(5 \mathrm{~mL})$. After stirring at r.t. for $16 \mathrm{~h}$, the reaction was quenched with $\mathrm{H}_{2} \mathrm{O}$ and extracted with EtOAc $(4 \times 25 \mathrm{~mL})$. The combined extracts were washed with $\mathrm{H}_{2} \mathrm{O}$, brine and dried (anh. $\left.\mathrm{Na}_{2} \mathrm{SO}_{4}\right)$. PLC (1:3:6; EtOAc/ $\mathrm{CH}_{2} \mathrm{Cl}_{2} /$ hexanes, double run) gave cis-5a and trans-5a.

cis-5a. Pale yellow oil, yield 52\%, $191 \mathrm{mg}$. IR (neat): $v 1790 \mathrm{~s}, 1732 \mathrm{~s}, 1195 \mathrm{~s} \mathrm{~cm}^{-1}$. ${ }^{1} \mathrm{H}-\mathrm{NMR}$ $\left(\mathrm{CDCl}_{3}, 300 \mathrm{MHz}\right.$, determined for two isomers): $\delta$ 7.06-7.27 (m, 10H, $\left.\mathrm{PhH}\right), 6.74(\mathrm{~d}, J 15.8,1 \mathrm{H}$, $\mathrm{PhCH}), 6.73$ (d, J 15.8, 1H, PhCH), 6.29-6.18 (m, 2H, 2×CH), 5.24-5.12 (m, 2H, 2×CH), 4.84$4.70(\mathrm{~m}, 2 \mathrm{H}, 2 \times \mathrm{CH}), 3.30-3.18(\mathrm{~m}, 2 \mathrm{H}, 2 \times \mathrm{CH}), 3.08-2.80\left(\mathrm{~m}, 4 \mathrm{H}, 2 \times \mathrm{CH}_{2}\right), 2.08-1.94(\mathrm{~m}, 2 \mathrm{H}$, $2 \times \mathrm{CH}$ ) , 1.89-1.60, 1.60-1.32, 1.16-0.83, 0.82-0.72, 0.72-0.67 (each m, 34H, methine, methylene and methyl protons). ${ }^{13} \mathrm{C}-\mathrm{NMR}\left(75 \mathrm{MHz}, \mathrm{CDCl}_{3}\right.$, determined for two isomers): $\delta 174.1(\mathrm{CO})$, $174.0(\mathrm{CO}), 170.2(\mathrm{CO}), 170.0(\mathrm{CO}), 135.4(\mathrm{C}), 135.3(\mathrm{C}), 134.3(2 \times \mathrm{CH}), 133.8(2 \times \mathrm{CH}), 128.7$ $(2 \times \mathrm{CH}), 128.6(\mathrm{CH}), 128.5(\mathrm{CH}), 126.8(2 \times \mathrm{CH}), 126.7(2 \times \mathrm{CH}), 124.8(\mathrm{CH}), 124.6(\mathrm{CH}), 82.2$ $(\mathrm{CH}), 81.8(\mathrm{CH}), 76.1(\mathrm{CH}), 76.0(\mathrm{CH}), 47.4(\mathrm{CH}), 47.0(\mathrm{CH}), 46.9(\mathrm{CH}), 46.8(\mathrm{CH}), 40.8$ $\left(\mathrm{CH}_{2}\right), 40.7\left(\mathrm{CH}_{2}\right), 34.0\left(2 \times \mathrm{CH}_{2}\right), 32.3\left(\mathrm{CH}_{2}\right), 31.9\left(\mathrm{CH}_{2}\right), 31.4(2 \times \mathrm{CH}), 26.4(\mathrm{CH}), 26.1(\mathrm{CH})$, $23.3\left(\mathrm{CH}_{2}\right), 23.0\left(\mathrm{CH}_{2}\right), 21.9\left(2 \times \mathrm{CH}_{3}\right), 20.7\left(\mathrm{CH}_{3}\right), 20.6\left(\mathrm{CH}_{3}\right), 16.2\left(\mathrm{CH}_{3}\right), 15.8\left(\mathrm{CH}_{3}\right) . \mathrm{MS}, \mathrm{m} / z$, $(\%)=371(\mathrm{M}+\mathrm{H})^{+}$. HRMS Calcd. for $\mathrm{C}_{23} \mathrm{H}_{30} \mathrm{NaO}_{4}[\mathrm{M}+\mathrm{Na}]^{+}$: 393.2042. Found 393.2037.

trans-5a. White solid, yield $24 \%, 88 \mathrm{mg}, \mathrm{mp} 98-100{ }^{\circ} \mathrm{C}\left(\mathrm{CH}_{2} \mathrm{Cl}_{2} /\right.$ hexanes). IR (neat): $v 1789 \mathrm{~s}$, $1734 \mathrm{~s}, 1193 \mathrm{sm}^{-1} .{ }^{1} \mathrm{H}-\mathrm{NMR}\left(300 \mathrm{MHz}, \mathrm{CDCl}_{3}\right): \delta 7.36-7.14(\mathrm{~m}, 5 \mathrm{H}, \mathrm{PhH}), 6.66(\mathrm{~d}, J 15.8,1 \mathrm{H}$, $\mathrm{PhCH}$ ), 6.07 (dd, J 7.2, 15.8, 1H, CH), 5.26 (dd, J 7.2, 7.8, 1H, CH), 4.59 (dt, J 4.4, 10.9, 1H, $\mathrm{CH}), 3.53(\mathrm{ddd}, J 7.5,7.8,8.5,1 \mathrm{H}, \mathrm{CH}), 2.95(\mathrm{dd}, J 7.5,17.6,1 \mathrm{H}, \mathrm{CHH}), 2.66(\mathrm{dd}, J 8.5,17.6$, $1 \mathrm{H}, \mathrm{CH} H$ ), 1.88-1.76, 1.76-1.62, 1.62-1.42, 1.39-1.20, 1.00-0.50 (each m, 15H, methine, methylene and methyl protons), $0.47\left(d, J 6.9,3 \mathrm{H}, \mathrm{CH}_{3}\right) .{ }^{13} \mathrm{C}-\mathrm{NMR}\left(75 \mathrm{MHz}, \mathrm{CDCl}_{3}\right): \delta 174.7$ (CO), $169.0(\mathrm{CO}), 135.2(\mathrm{C}), 135.1(2 \times \mathrm{CH}), 128.6(2 \times \mathrm{CH}), 126.9(2 \times \mathrm{CH}), 121.6(\mathrm{CH}), 80.2$ $(\mathrm{CH}), 76.0(\mathrm{CH}), 46.5(\mathrm{CH}), 45.8(\mathrm{CH}), 41.0\left(\mathrm{CH}_{2}\right), 33.9\left(\mathrm{CH}_{2}\right), 31.4\left(\mathrm{CH}_{2}\right), 31.3(\mathrm{CH}), 26.0$ $(\mathrm{CH}), 22.9\left(\mathrm{CH}_{2}\right), 21.8\left(\mathrm{CH}_{3}\right), 20.7\left(\mathrm{CH}_{3}\right), 15.7\left(\mathrm{CH}_{3}\right) . \mathrm{MS}, m / z,(\%)=393(\mathrm{M}+\mathrm{Na})^{+}$. HRMS Calcd. for $\mathrm{C}_{23} \mathrm{H}_{30} \mathrm{NaO}_{4}[\mathrm{M}+\mathrm{Na}]^{+}:$393.2042. Found: 393.2055. 


\section{(2-Isopropyl-5-methylcyclohexyl)-5-oxo-2-(3,4,5-trimethoxyphenyl)tetrahydrofuran-3-}

carboxylate (5b). Treatment of 2 generated from 1 (1 mmol) with $\mathrm{ZnCl}_{2}(2 \mathrm{mmol})$ and 3,4,5trimethoxybenzaldehyde $(196 \mathbf{~ m g}, 1 \mathbf{~ m m o l})$ followed by lactonization gave cis-5b and trans-5b. cis-5b. Pale yellow gum, yield 59\%, $216 \mathrm{mg}$. IR (neat): $v$ 1785s, 1725s, 1595s, 1464s, 1192s, $1131 \mathrm{~s} \mathrm{~cm}^{-1} .{ }^{1} \mathrm{H}-\mathrm{NMR}\left(500 \mathrm{MHz}, \mathrm{CDCl}_{3}\right.$, determined for two isomers): $\delta 6.49$ (s, 2H, $\mathrm{ArH}$ ), 6.48 (s, 2H, ArH), $5.50(\mathrm{~d}, J 7.7,1 \mathrm{H}, \mathrm{CH}), 5.49$ (d, J 7.8, 1H, CH), 4.70 (dt, J 4.4, 11.1, 1H, CH), 4.67 $(\mathrm{dt}, J 4.5,11.1,1 \mathrm{H}, \mathrm{CH}), 3.79\left(\mathrm{~s}, 6 \mathrm{H}, 2 \times \mathrm{OCH}_{3}\right), 3.78\left(\mathrm{~s}, 6 \mathrm{H}, 2 \times \mathrm{OCH}_{3}\right), 3.77\left(\mathrm{~s}, 3 \mathrm{H}, \mathrm{OCH}_{3}\right), 3.76$ $\left(\mathrm{s}, 3 \mathrm{H}, \mathrm{OCH}_{3}\right), 3.30-3.20(\mathrm{~m}, 2 \mathrm{H}, 2 \times \mathrm{CH}), 2.95-2.80\left(\mathrm{~m}, 4 \mathrm{H}, 2 \times \mathrm{CH}_{2}\right), 1.95-1.87(\mathrm{~m}, 2 \mathrm{H}), 1.75-$ $1.67(\mathrm{~m}, 1 \mathrm{H}), 1.65-1.50(\mathrm{~m}, 5 \mathrm{H}), 1.47-1.35(\mathrm{~m}, 2 \mathrm{H}), 1.35-1.25(\mathrm{~m}, 2 \mathrm{H}), 1.05-0.86(\mathrm{~m}, 4 \mathrm{H}), 0.85-$ $0.80(\mathrm{~m}, 11 \mathrm{H}), 0.75\left(\mathrm{~d}, J 7.0,3 \mathrm{H}, \mathrm{CH}_{3}\right), 0.68\left(\mathrm{~d}, J 7.0,3 \mathrm{H}, \mathrm{CH}_{3}\right), 0.62\left(\mathrm{~d}, J 7.0,3 \mathrm{H}, \mathrm{CH}_{3}\right) .{ }^{13} \mathrm{C}-$ NMR (125 MHz, $\mathrm{CDCl}_{3}$, determined for two isomers): $\delta 174.0(\mathrm{CO}), 173.9(\mathrm{CO}), 170.3(\mathrm{CO})$, $170.2(\mathrm{CO}), 153.6(2 \times \mathrm{C}), 153.5(2 \times \mathrm{C}), 133.6(2 \times \mathrm{C}), 133.3(2 \times \mathrm{C}), 102.5(2 \times \mathrm{CH}), 102.3(2 \times \mathrm{CH})$, $82.4(\mathrm{CH}), 82.3(\mathrm{CH}), 76.0(\mathrm{CH}), 75.9(\mathrm{CH}), 60.7\left(2 \times \mathrm{OCH}_{3}\right), 56.1\left(4 \times \mathrm{OCH}_{3}\right), 49.1(\mathrm{CH}), 49.0$ $(\mathrm{CH}), 46.8(\mathrm{CH}), 46.7(\mathrm{CH}), 40.8\left(\mathrm{CH}_{2}\right), 40.7\left(\mathrm{CH}_{2}\right), 33.9\left(2 \times \mathrm{CH}_{2}\right), 32.7\left(\mathrm{CH}_{2}\right), 32.4\left(\mathrm{CH}_{2}\right), 31.2$ $(2 \times \mathrm{CH}), 26.3(\mathrm{CH}), 26.2(\mathrm{CH}), 23.2\left(\mathrm{CH}_{2}\right), 23.1\left(\mathrm{CH}_{2}\right), 21.8\left(2 \times \mathrm{CH}_{3}\right), 20.6\left(\mathrm{CH}_{3}\right), 20.5\left(\mathrm{CH}_{3}\right)$, $16.1\left(\mathrm{CH}_{3}\right), 16.0\left(\mathrm{CH}_{3}\right)$. MS, $m / z,(\%)=435(\mathrm{M}+\mathrm{H})^{+}$. HRMS Calcd. for $\mathrm{C}_{24} \mathrm{H}_{35} \mathrm{O}_{7}[\mathrm{M}+\mathrm{H}]^{+}$: 435.2383. Found: 435.2367.

trans-5b. Pale yellow gum, yield 12\%, $50 \mathrm{mg}$. IR (neat): $v 1785 s, 1725 s, 1595 s, 1509 s, 1464 s$, $1199 s, 1131 \mathrm{~s} \mathrm{~cm}^{-1}$. ${ }^{1} \mathrm{H}-\mathrm{NMR}\left(300 \mathrm{MHz}, \mathrm{CDCl}_{3}\right.$, determined for two isomers): $\delta 6.47$ (s, $2 \mathrm{H}$, $\operatorname{ArH}), 6.45(\mathrm{~s}, 2 \mathrm{H}, \mathrm{ArH}), 5.60(\mathrm{~d}, J 7.4,2 \mathrm{H}, 2 \times \mathrm{CH}), 4.40-4.25(\mathrm{~m}, 2 \mathrm{H}, 2 \times \mathrm{CH}), 3.79(\mathrm{~s}, 12 \mathrm{H}$, $\left.4 \times \mathrm{OCH}_{3}\right), 3.76\left(\mathrm{~s}, 6 \mathrm{H}, 2 \times \mathrm{OCH}_{3}\right), 3.70-3.55(\mathrm{~m}, 2 \mathrm{H}, 2 \times \mathrm{CH}), 3.05-2.70\left(\mathrm{~m}, 4 \mathrm{H}, 2 \times \mathrm{CH}_{2}\right), 1.60-$ $1.30(\mathrm{~m}, 6 \mathrm{H}), 1.30-1.00(\mathrm{~m}, 6 \mathrm{H}), 0.95-0.80(\mathrm{~m}, 18 \mathrm{H}), 0.52\left(\mathrm{~d}, J 6.8,3 \mathrm{H}, \mathrm{CH}_{3}\right), 0.40(\mathrm{~d}, J 6.8,3 \mathrm{H}$, $\left.\mathrm{CH}_{3}\right) .{ }^{13} \mathrm{C}-\mathrm{NMR}\left(125 \mathrm{MHz}, \mathrm{CDCl}_{3}\right.$, determined for two isomers): $\delta 174.8(\mathrm{CO}), 174.6(\mathrm{CO})$, $169.6(\mathrm{CO}), 169.4(\mathrm{CO}), 153.3(4 \mathrm{xC}), 138.2(\mathrm{C}), 138.0(\mathrm{C}), 130.5(2 \mathrm{xC}), 103.1(2 \mathrm{xCH}), 102.8$ $(2 \times \mathrm{CH}), 81.3(\mathrm{CH}), 81.2(\mathrm{CH}), 75.5(\mathrm{CH}), 75.4(\mathrm{CH}), 60.8\left(\mathrm{OCH}_{3}\right), 60.7\left(\mathrm{OCH}_{3}\right), \quad 56.1$ $\left(2 \mathrm{xOCH}_{3}\right), 56.0\left(2 \mathrm{xOCH}_{3}\right), 46.8(\mathrm{CH}), 46.6(\mathrm{CH}), 46.5(\mathrm{CH}), 46.1(\mathrm{CH}), 40.4\left(\mathrm{CH}_{2}\right), 39.9\left(\mathrm{CH}_{2}\right)$, $33.9\left(2 \mathrm{xCH}_{2}\right), 33.0\left(\mathrm{CH}_{2}\right), 32.4\left(\mathrm{CH}_{2}\right), 31.1(\mathrm{CH}), 31.0(\mathrm{CH}), 25.9(\mathrm{CH}), 25.6(\mathrm{CH}), 23.0\left(\mathrm{CH}_{2}\right)$, $22.7\left(\mathrm{CH}_{2}\right), 21.8\left(\mathrm{CH}_{3}\right), 21.7\left(\mathrm{CH}_{3}\right), 20.6\left(\mathrm{CH}_{3}\right), 20.5\left(\mathrm{CH}_{3}\right), 16.1\left(\mathrm{CH}_{3}\right), 16.0\left(\mathrm{CH}_{3}\right)$. HRMS Calcd. for $\mathrm{C}_{24} \mathrm{H}_{34} \mathrm{NaO}_{7}[\mathrm{M}+\mathrm{Na}]^{+}:$457.2202. Found: 457.2219.

(2-Isopropyl-5-methylcyclohexyl)-4-((E)-1-hydroxy-3-phenylallyl)-5-oxo-2-styryltetra-

hydrofuran-3-carboxylate (6). White solid, yield 56\%, $281 \mathrm{mg}, \mathrm{mp} \quad 188-189{ }^{\circ} \mathrm{C}$ $\left(\mathrm{CH}_{2} \mathrm{Cl}_{2}\right.$ /hexanes), $[\alpha]^{24}-41^{\circ}\left(c 1, \mathrm{CH}_{2} \mathrm{Cl}_{2}\right)$. IR (KBr): v 3531br, 1794s, 1728s, 1323m, 1178s, $1165 \mathrm{sm}^{-1} .{ }^{1} \mathrm{H}-\mathrm{NMR}\left(300 \mathrm{MHz}, \mathrm{CDCl}_{3}\right): \delta 7.46-7.19(\mathrm{~m}, 10 \mathrm{H}, \mathrm{PhH}), 6.77(\mathrm{~d}, J 15.7,1 \mathrm{H}$, $\mathrm{PhCH}$ ), 6.71 (d, J 15.7, 1H, PhCH), 6.25 (dd, J 8.2, 15.8, 1H, CH), 6.17 (dd, J 5.0, 15.9, 1H, $\mathrm{CH}), 5.25-5.03(\mathrm{~m}, 1 \mathrm{H}, \mathrm{CH}), 4.97(\mathrm{t}, J 8.2,1 \mathrm{H}, \mathrm{CH}), 4.56(\mathrm{dt}, J 4.2,10.9,1 \mathrm{H}, \mathrm{CH}), 3.55-3.40(\mathrm{~m}$, $2 \mathrm{H}, 2 \times \mathrm{CH}), 2.60-2.45$ (br s, $1 \mathrm{H}, \mathrm{OH}), 1.801 .60(\mathrm{~m}, 2 \mathrm{H}, 2 \times \mathrm{CH}), 1.60-1.48(\mathrm{~m}, 3 \mathrm{H}, 3 \times \mathrm{CH})$, 1.35-1.13 (m, 1H, CH), 1.00-0.80 (m, 1H, CHH), 0.75-0.62 (m, 1H, CHH), 0.62-0.48 (m, 10H, $\mathrm{CHH}$ and $\left.3 \times \mathrm{CH}_{3}\right) .{ }^{13} \mathrm{C}-\mathrm{NMR}\left(75 \mathrm{MHz}, \mathrm{CDCl}_{3}\right): \delta 174.9(\mathrm{CO}), 170.8(\mathrm{CO}), 136.1(\mathrm{C}), 135.7$ $(\mathrm{CH}), 135.2(\mathrm{C}), 131.3(\mathrm{CH}), 128.7(\mathrm{CH}), 128.6(4 \times \mathrm{CH}), 128.0(\mathrm{CH}), 127.5(\mathrm{CH}), 126.9$ $(2 \times \mathrm{CH}), 126.6,(2 \times \mathrm{CH}), 124.2(\mathrm{CH}), 81.7(\mathrm{CH}), 76.2(\mathrm{CH}), 68.9(\mathrm{CH}), 51.3(\mathrm{CH}), 47.7(\mathrm{CH})$, 
$46.5(\mathrm{CH}), 40.2\left(\mathrm{CH}_{2}\right), 33.9\left(\mathrm{CH}_{2}\right), 31.1(\mathrm{CH}), 25.6(\mathrm{CH}), 22.7\left(\mathrm{CH}_{2}\right), 21.7\left(\mathrm{CH}_{3}\right), 20.4\left(\mathrm{CH}_{3}\right)$, $15.3\left(\mathrm{CH}_{3}\right)$. MS, $m / z,(\%)=525(\mathrm{M}+\mathrm{Na})^{+}$. HRMS Calcd. for $\mathrm{C}_{32} \mathrm{H}_{38} \mathrm{NaO}_{5}[\mathrm{M}+\mathrm{Na}]^{+}: 525.2617$. Found: 525.2626.

Bis(2-isopropyl-5-methylcyclohexyl) 2-benzylsuccinate (8). Dienolate 2 derived from 1 (1 mmol) was treated with a solution of benzyl bromide $(178 \mathrm{mg}, 1 \mathrm{mmol})$ in $\mathrm{THF}(2 \mathrm{~mL})$ at $-78^{\circ} \mathrm{C}$ for $2 \mathrm{~h}$. The reaction mixture was quenched with a saturated $\mathrm{NH}_{4} \mathrm{Cl}$ solution at $-78{ }^{\circ} \mathrm{C}$, warmed up to r.t., and extracted with EtOAc $(3 \times 10 \mathrm{~mL})$. CC $(1 \%$ EtOAc in hexane $)$ gave 8. Colorless viscous oil, yield $89 \%, 435 \mathrm{mg}, \mathrm{dr}=1: 1.2$ (determined by ${ }^{13} \mathrm{C}-\mathrm{NMR}$ (without NOE) spectrum); IR (neat): v 2956s, 2870s, 1732s, 1456s, 1370s, 1175s, 1149s cm ${ }^{-1} .{ }^{1} \mathrm{H}-\mathrm{NMR}\left(300 \mathrm{MHz}, \mathrm{CDCl}_{3}\right.$, determined for two isomers): $\delta$ 7.24-7.07 (m, $10 \mathrm{H}, \mathrm{PhH}), 4.65-4.50(\mathrm{~m}, 4 \mathrm{H}, 4 \times \mathrm{CH}), 3.11-2.90$ (m, 4H, $2 \times \mathrm{CHCHHPh),} \mathrm{2.75-2.63} \mathrm{(m,} \mathrm{2H,} 2 \times \mathrm{CH} H \mathrm{Ph}), 2.61-2.50(\mathrm{~m}, 2 \mathrm{H}, 2 \times \mathrm{CHH}), 2.36-2.22$ $(\mathrm{m}, 2 \mathrm{H}, 2 \times \mathrm{CH} H$ ), 1.94-1.68, 1.64-1.20, 1.03-0.61, 0.59-0.52 (each $\mathrm{m}, 72 \mathrm{H}$, methine, methylene and methyl protons). ${ }^{13} \mathrm{C}-\mathrm{NMR}\left(75 \mathrm{MHz}, \mathrm{CDCl}_{3}\right.$, determined for two isomers): $\delta 173.8(\mathrm{CO})$, $173.6(\mathrm{CO}), 171.25(\mathrm{CO}), 171.23(\mathrm{CO}), 138.4(\mathrm{C}), 138.3(\mathrm{C}), 129.1(2 \times \mathrm{CH}), 129.0(2 \times \mathrm{CH})$, $128.4(4 \times \mathrm{CH}), 126.52(\mathrm{CH}), 126.51(\mathrm{CH}), 74.5(\mathrm{CH}), 74.43(\mathrm{CH}), 74.39(\mathrm{CH}), 74.28(\mathrm{CH})$, $46.91(\mathrm{CH}), 46.87(\mathrm{CH}), 46.85(\mathrm{CH}), 46.76(\mathrm{CH}), 43.4(\mathrm{CH}), 43.0(\mathrm{CH}), 40.8\left(2 \times \mathrm{CH}_{2}\right), 40.6$ $\left(\mathrm{CH}_{2}\right), 40.5\left(\mathrm{CH}_{2}\right), 37.74\left(\mathrm{CH}_{2}\right), 37.70\left(\mathrm{CH}_{2}\right), 35.8\left(\mathrm{CH}_{2}\right), 35.2\left(\mathrm{CH}_{2}\right), 34.21\left(2 \times \mathrm{CH}_{2}\right), 34.19$ $\left(2 \times \mathrm{CH}_{2}\right), 31.33(2 \times \mathrm{CH}), 31.31(\mathrm{CH}), 31.28(\mathrm{CH}), 26.2(\mathrm{CH}), 26.1(\mathrm{CH}), 25.9(\mathrm{CH}), 25.7(\mathrm{CH})$, $23.4\left(\mathrm{CH}_{2}\right), 23.3\left(\mathrm{CH}_{2}\right), 23.2\left(\mathrm{CH}_{2}\right), 23.0\left(\mathrm{CH}_{2}\right), 22.0\left(\mathrm{CH}_{3}\right), 21.99\left(\mathrm{CH}_{3}\right), 21.97\left(\mathrm{CH}_{3}\right), 21.96$ $\left(\mathrm{CH}_{3}\right), 20.81\left(\mathrm{CH}_{3}\right), 20.80\left(\mathrm{CH}_{3}\right), 20.76\left(\mathrm{CH}_{3}\right), 20.7\left(\mathrm{CH}_{3}\right), 16.3\left(\mathrm{CH}_{3}\right), 16.2\left(\mathrm{CH}_{3}\right), 16.1\left(\mathrm{CH}_{3}\right)$, $15.9\left(\mathrm{CH}_{3}\right)$. MS, $m / z,(\%)=484\left(\mathrm{M}^{+}\right)$. Anal. Calcd. for $\mathrm{C}_{31} \mathrm{H}_{48} \mathrm{O}_{4}(484.36)$ : C, 76.82; $\mathrm{H}, 9.98 \%$. Found: C, 76.93; H, 10.24\%.

Bis(2-isopropyl-5-methylcyclohexyl)-2,3-dibenzylsuccinate (9). White solid, yield 7\%, $41 \mathrm{mg}$, mp 134-139 ${ }^{\circ} \mathrm{C}$ (EtOAc/hexanes). IR (nujol): $v$ 1740s, 1723s, 1197s, 1163s, 1148s cm ${ }^{-1} .{ }^{1} \mathrm{H}-$ NMR (300 MHz, $\mathrm{CDCl}_{3}$, determined as mixtures of isomers): $\delta 7.30-7.05(\mathrm{~m}, 20 \mathrm{H}, \mathrm{PhH}), 4.70$ $4.57(\mathrm{~m}, 4 \mathrm{H}, 4 \times \mathrm{CH}), 3.15-2.90(\mathrm{~m}, 12 \mathrm{H}), 2.07-1.92,1.90-1.52,1.52-1.18,1.12-0.58$ (each m, $72 \mathrm{H}$, methine, methylene and methyl protons). ${ }^{13} \mathrm{C}-\mathrm{NMR}\left(75 \mathrm{MHz}, \mathrm{CDCl}_{3}\right.$, determined as mixtures of isomers): $\delta 172.8(2 \times \mathrm{CO}), 172.7(2 \times \mathrm{CO}), 139.1(2 \times \mathrm{C}), 139.0(2 \times \mathrm{C}), 129.1(\mathrm{CH})$, $129.0(\mathrm{CH}), 128.9(\mathrm{CH}), 128.4(\mathrm{CH}), 128.3(\mathrm{CH}), 126.3(\mathrm{CH}), 74.6(2 \times \mathrm{CH}), 74.5(2 \times \mathrm{CH}), 48.6$ $(2 \times \mathrm{CH}), 48.0(2 \times \mathrm{CH}), 46.8(2 \times \mathrm{CH}), 40.8\left(2 \times \mathrm{CH}_{2}\right), 40.6\left(2 \times \mathrm{CH}_{2}\right), 35.3\left(2 \times \mathrm{CH}_{2}\right), 34.9\left(2 \times \mathrm{CH}_{2}\right)$, $34.2\left(2 \times \mathrm{CH}_{2}\right), 34.1\left(2 \times \mathrm{CH}_{2}\right), 31.4(2 \times \mathrm{CH}), 31.3(2 \times \mathrm{CH}), 25.7(2 \times \mathrm{CH}), 25.6(2 \times \mathrm{CH}), 23.1$ $\left(2 \times \mathrm{CH}_{2}\right), 23.0\left(2 \times \mathrm{CH}_{2}\right), 22.0\left(2 \times \mathrm{CH}_{3}\right), 21.9\left(2 \times \mathrm{CH}_{3}\right), 20.9\left(2 \times \mathrm{CH}_{3}\right), 20.8\left(2 \times \mathrm{CH}_{3}\right), 16.0$ $\left(2 \times \mathrm{CH}_{3}\right), 15.9\left(2 \times \mathrm{CH}_{3}\right) . \mathrm{MS}, \mathrm{m} / z,(\%)=576(\mathrm{M}+1)^{+}, 575\left(\mathrm{M}^{+}\right)$. HRMS Calcd. for $\mathrm{C}_{38} \mathrm{H}_{54} \mathrm{NaO}_{4}$ $[\mathrm{M}+\mathrm{Na}]^{+}:$597.3920. Found: 597.3969.

\section{Acknowledgements}

We acknowledge financial supports from Mahidol University (to D.S.), the Thailand Research Fund (BRG5380019), the Office of the Higher Education Commission and Mahidol University 
under the National Research Universities Initiative, and the Center of Excellence for Innovation in Chemistry (PERCH-CIC).

\section{References and Notes}

1. See, for examples; (a) Csákÿ, A. G.; Plumet, J. Chem. Soc. Rev. 2001, 30, 313. (b) Sibi, M. P.; Liu, P.; Ji, J.; Hajra, S.; Chen, J. J. Org. Chem. 2002, 67, 1738. (c) Sibi, M. P.; Hasegawa, H. Org. Lett. 2002, 4, 3347. (d) Langer, T.; Illich, M.; Helmchen, G. Synlett 1996, 1137. (e) Beckett, R. P.; Crimmin, M. J.; Davis, M. H.; Spavold, Z. Synlett 1993, 137. (f) Kim, H.-C.; Park, O.-S. Tetrahedron: Asymmetry 2008, 19, 896. (g) Hajra, S.; Karmakar, A.; Giri, A. K.; Hazra, S. Tetrahedron Lett. 2008, 49, 3625. (h) Patel R. M.; Argade, N. P. Synthesis 2010, 1188. (i) Wang, X.; Lin, J.; Chen, Y.; Zhong, W.; Zhao, G.; Liu, H.; Li, S.; Wang, L.; Li, S. Bioorg. Med. Chem. 2009, 17, 1898.

2. (a) Long, N. R.; Rathke, M. W. Synth. Commun. 1981, 11, 687. (b) Mahalanabis, K. K.; Mumtaz, M.; Snieckus, V. Tetrahedron Lett. 1982, 23, 3971. (c) Mahalanabis, K. K.; Mumtaz, M.; Snieckus, V. Tetrahedron Lett. 1982, 23, 3975. (d) Furuta, K.; Misumi, A.; Mori, A.; Ikeda, N.; Yamamoto, H. Tetrahedron Lett. 1984, 25, 669. (e) Furuta, K.; Ikeda, N.; Yamamoto, H. Tetrahedron Lett. 1984, 25, 675. (f) Garratt, P. J.; Zahler, R. Tetrahedron Lett. 1979, 20, 73. (g) Bilyard, K. G.; Garratt, P. J.; Underwood, A. J.; Zahler, R. Tetrahedron Lett. 1979, 20, 1815. (h) Garratt, P. J.; Porter, J. R. Tetrahedron Lett. 1987, 28, 351. (i) Garratt, P. J.; Pielke, M.; Porter, J. R. Tetrahedron Lett. 1987, 28, 589. (j) Doecke, C. W.; Garratt, P. J. J. Chem. Soc.,Chem . Commun. 1981, 873. (k) Bilyard, K. G.; Garratt, P. J. Tetrahedron Lett. 1981, 22, 1755. (1) Jun, J.-G.; Mundy, B. P. Bull. Korean Chem. Soc. 1987, 8, 310. (m) Corey, E. J.; Su, W.-G. Tetrahedron Lett. 1987, 28, 5241. (n) Girard, C.; Bloch, R. Tetrahedron Lett. 1982, 23, 3683. (o) Noire, P. D.; Franck, R. W. Tetrahedron Lett. 1982, 23, 1031. (p) Pohmakotr, M.; Reutrakul, V.; Phongpradit, T.; Chansri, A. Chem. Lett. 1982, 687.

3. Sibi, M. P.; Deshpande, P. K.; La Loggia, A. J. Synlett 1996, 343, and references cited therein.

4. (a) Pohmakotr, M.; Harnying, W.; Tuchinda, P.; Reutrakul, V. Helv. Chim. Acta 2002, 85, 3792. (b) Pohmakotr, M.; Sampaongoen, L.; Issaree, A.; Tuchinda, P.; Reutrakul, V. Tetrahedron Lett. 2003, 44, 6717. (c) Pohmakotr, M.; Issaree, A.; Sampaongoen, L.; Tuchinda, P.; Reutrakul, V. Tetrahedron Lett. 2003, 44, 7937. (d) Pohmakotr, M.; Komutkul, T.; Tuchinda, P.; Prabpai, S.; Kongsaeree, P.; Reutrakul, V. Tetrahedron 2005, 61, 5311. (e) Pohmakotr, M.; Pinsa, A.; Mophuang, T.; Tuchinda, P.; Prabpai, S.; Kongsaeree, P.; Reutrakul, V. J. Org. Chem. 2006, 71, 386.

5. (a) Ayres, D. C.; Loike, J. D. Lignans: Chemical, Biological and Clinical Properties; Cambridge University Press: Cambridge, 1990. (b) Ward, R. S. Nat. Prod. Rep. 1999, 16, 75. 
6. (a) Pohmakotr, M.; Soorukram, D.; Tuchinda, P.; Prabpai, S.; Kongsaeree, P.; Reutrakul, V. Tetrahedron Lett. 2004, 45, 4315. (b) Jung, J.-C.; Kim, J.-C.; Moon, H.-I.; Park, O.-S.; Tetrahedron Lett. 2006, 47, 6433. (c) Kim, J.-C.; Kim, K.-H.; Jung, J.-C.; Park, O.-S.; Tetrahedron: Asymmetry 2006, 17, 3. (d) Evans, D. A.; Takacs, J. M. Tetrahedron Lett. 1980, 21, 4233.

7. Misumi, A.; Iwanaga, K.; Furuta, K.; Yamamoto, H. J. Am. Chem. Soc. 1985, 107, 3343.

8. (a) Lee, E.; Choi, S. J.; Kim, H.; Han, H. O.; Kim, Y. K.; Min, S. J.; Son, S. H.; Lim, S. M.; Jang, W. S. Angew. Chem. Int., Ed. 2002, 41, 176. (b) Trost, B. M.; Dirat, O.; Gunzner, J. L. Angew. Chem. Int., Ed. 2002, 41, 841. (c) Kende, A. S.; Fujii, Y.; Mendoza, J. S. J. Am. Chem. Soc. 1990, 112, 9645. (d) Myers, M. C.; Witschi, M. A.; Larionova, N. V.; Franck, J. M.; Haynes, R. D.; Hara, T.; Grajkowski, A.; Appella, D. H. Org. Lett. 2003, 5, 2695. (e) Pokorski, J. K.; Myers, M. C.; Appella, D. H. Tetrahedron Lett. 2005, 46, 915.

9. Furuta, K.; Iwanaga, K, K.; Yamamoto, H. Org. Synth. 1993, Coll. Vol. 8, 141.

10. In general, it was widely assumed that $Z$ enolates tend to give syn aldols and $E$ enolates tend to give anti aldols. Moreover, greater anti selectivity was usually observed in the zincmediated aldol reactions. See; Heathcock, C. H. In Asymmetric Synthesis; Morrison, J. D. Ed.; Academic Press: Orlando, 1984, Vol. 3, p 111.

11. Hydrolysis of trans-5a using $\mathrm{LiOH}$ in THF at room temperature gave the corresponding trans-paraconic acid, whereas hydrolysis of cis-5a under similar reaction conditions led to partial racemization providing cis- and trans-paraconic acids.

12. The diastereomeric ratio of compound $\mathbf{8}$ was determined from the ${ }^{13} \mathrm{C}-\mathrm{NMR}$ (without NOE) spectra. 